\title{
Task Synchronization in Reservation-Based Real-Time Systems
}

\author{
Giuseppe Lipari, Member, IEEE, Gerardo Lamastra, Member, IEEE, and \\ Luca Abeni, Member, IEEE
}

\begin{abstract}
In this paper, we present the BandWidth Inheritance (BWI) protocol, a new strategy for scheduling real-time tasks in dynamic systems, which extends the resource reservation framework to systems where tasks can interact through shared resources. The proposed protocol provides temporal isolation between independent groups of tasks and enables a schedulability analysis for guaranteeing the performance of hard real-time tasks. We show that BWI is the natural extension of the well-known Priority Inheritance Protocol to dynamic reservation systems. A formal analysis of the protocol is presented and a guarantee test for hard real-time tasks is proposed that takes into account the case in which hard real-time tasks interact with soft real-time tasks.
\end{abstract}

Index Terms—Real-time scheduling, dynamic system, resource reservation, priority inheritance, constant bandwidth server.

\section{INTRODUCTION}

$\mathrm{T}$ HE main goal of a real-time scheduler is to provide temporal guarantees. In a hard real-time system, the scheduler must guarantee that, under certain worst-case assumptions, the temporal constraints of all tasks are respected. When mixing hard, soft, and non-real-time tasks, providing such temporal guarantees becomes a complex problem. In dynamic real-time systems, tasks can be activated dynamically and the system has no a priori knowledge about their run-time behavior. Classical real-time schedulability analyses are not appropriate for dynamic real-time systems because they require a priori knowledge of the characteristics of all the tasks to guarantee that every hard real-time task will meet its deadline.

The resource reservation framework is a class of techniques that have been proven very effective in jointly scheduling hard real-time (HRT) and soft real-time (SRT) tasks. In particular, these approaches provide 1) temporal isolation between tasks and 2) schedulability analysis for HRT tasks. However, tasks are assumed to be independent. This is a severe limitation that hinders their utilization in real operating systems. The extension of the underlying model to cope with tasks that access shared resources through mutually exclusive (mutex) semaphores has only recently been addressed [1], [2], [3].

In this paper, a new protocol, BandWidth Inheritance (BWI), is presented. It extends the Constant Bandwidth Server (CBS) algorithm [4] to real-time tasks that can access shared resources via critical sections, by using a technique derived from the Priority Inheritance Protocol (PIP) [5].

- G. Lipari is with the Retis Lab, Scuola Superiore Sant'Anna, piazza Martiri della Libertà 33, 56100, Pisa (PI), Italy. E-mail: lipari@sssup.it.

- G. Lamastra is with Telecom Italia Lab, G. Reiss Romoli 274, 10148 Torino (TO), Italy. E-mail: Gerardo.Lamastra@tilab.com.

- L. Abeni is with Broadsat S.r.l., viale Garibaldi 54, 51017, Pescia (PT), Italy. E-mail: lucabe72@email.it.

Manuscript received 11 June 2002; revised 17 Mar. 2003; accepted 27 May 2004.

For information on obtaining reprints of this article, please send e-mail to: tc@computer.org, and reference IEEECS Log Number 116736.
In the remainder of this paper, we describe the BWI protocol and its properties. Then, we provide a schedulability analysis for HRT tasks. This protocol does not require any a priori knowledge of each task's behavior. Only the analysis is based on the knowledge of the worstcase behavior of the HRT tasks. Hence, the BWI protocol is suitable for a real operating system.

\section{Related Work}

Deng and Liu [6] proposed a model of dynamic real-time systems called the open system model. In their model, an application is a set of tasks and a server (i.e., an algorithm of the class of the aperiodic server algorithms [7], [8], [9]) is used to handle each different application. A customized scheduler can be associated with each server in a hierarchical way. However, the algorithms proposed by Deng and Liu require a priori knowledge of the tasks' execution times even for SRT tasks.

A better approach is to provide temporal isolation between tasks such that each task is protected from the misbehaviors of the other tasks. This property is also called Bandwidth Isolation (BIP) [4], [10]. The net effect is that each task executes as though it were executing on a slower dedicated processor. The BIP can be implemented using the resource reservation framework [11], [12]. In this framework, when a task arrives in the system requiring a certain level of Quality of Service (QoS), an admission test is run. If, given the current system load, the required level of QoS can be guaranteed, the task is accepted in the system and assigned a certain fraction of the system resources. Many scheduling algorithms based on the resource reservation framework have been proposed. The temporal isolation and the resource reservation concepts were formally introduced for the first time in the RT-Mach operating system [11].

The Resource Kernel (RK) [12] is the most complete work to explicitly address the temporal isolation problem. An RK allows each task to reserve a hardware resource for a certain amount of time $C$ every interval of time of length $T$. The RK 
technology is particularly interesting since it seamlessly provides QoS guarantees even to non-real-time legacy applications and it has been recently ported to Linux [13].

Resource-reservation techniques, originally based on a fixed-priority scheme, have also been applied to dynamic priority schemes like the Earliest Deadline First (EDF) scheduler [14], [4]. One of the advantages of using EDF is that it is an optimal scheduler and permits very high processor utilization [15]. The Constant Bandwidth Server (CBS) [4] is based on EDF and uses a deadline postponing mechanism to efficiently provide the BIP. In particular, the CBS algorithm enables a schedulability analysis for HRT tasks and probabilistic analysis for SRT tasks.

All the algorithms cited so far support only independent tasks. This is a major limitation for their implementation in an operating system. In the real world, many real-time tasks communicate by means of shared memory protected by mutex semaphores.

Many researchers addressed the problem of providing guarantees to hard real-time tasks accessing shared resources. It has been proven that, if classical mutex semaphores are used, a particular problem arises, known as priority inversion. This problem was first described by Sha et al. [5], who proposed two solutions, the Priority Inheritance Protocol (PIP) and the Priority Ceiling Protocol (PCP). Similar protocols have been proposed for dynamic priority schedulers [16], [17], [18].

Applying these techniques to resource reservations is not trivial. For example, the CBS algorithm assumes a very simple task model, where no task can suspend itself or block on a critical section. If tasks are allowed to suspend, the properties of the CBS algorithm are not valid anymore (see Section 4 for more details).

Kuo and Li [19] propose an extension to the open system model presented in [6] that accounts for shared resources. In [1], it is shown how to combine the Stack Resource Policy (SRP) [17] with aperiodic servers, in order to share resources between aperiodic tasks and hard real-time tasks. This approach has been recently applied to the CBS algorithm [2]. However, these solutions require a priori knowledge of the maximum resource usage time for each task; otherwise, the protocol may not work properly. This information may not be available in dynamic real-time systems.

The problem of priority inversion in reservation-based systems was also considered by De Niz et al. [3]. The reserve-inheritance approach proposed therein resembles the approach proposed in this paper. However, while their methodology is based on fixed priority scheduling, our protocol is based on dynamic priority scheduling. In addition, in this paper, we propose a new sufficient schedulability analysis for HRT tasks, even when they interact with SRT tasks.

\section{SYSTEM MODEL}

\subsection{Definitions}

A real-time task $\tau_{i}$ is a stream of jobs, or instances, $J_{i, j}$ (where $J_{i, j}$ is the $j$ th job of task $\left.\tau_{i}\right)$; each job is a request for execution on a shared processor and is characterized by an arrival time $a_{i, j}$, an execution time $c_{i, j}$, and an absolute deadline $d_{i, j}$. A real-time task is also assigned a relative deadline $D_{i}$ and $d_{i, j}=a_{i, j}+D_{i}$. We denote the finishing time of job $J_{i, j}$ with $f_{i, j}$.

Real-time tasks can be hard or soft. A task $\tau_{i}$ is said to be a hard real-time (HRT) task if all its jobs must complete within their deadline $\left(\forall j f_{i, j} \leq d_{i, j}\right)$; otherwise, a critical failure may occur in the system. HRT tasks need to be guaranteed a priori. In order to perform a schedulability test, every HRT task $\tau_{i}$ is characterized by a minimum interarrival time $T_{i}=\min _{j}\left\{a_{i, j+1}-a_{i, j}\right\}$ and a worst-case execution time (WCET) $C_{i}=\max _{j}\left\{c_{i, j}\right\}$.

A task is said to be a soft real-time (SRT) task if it can tolerate an occasional deadline miss. Usually, it is difficult to compute the WCET for an SRT task. Moreover, in most cases, the WCET of an SRT task is much higher than its average-case execution time. Therefore, to allocate the processor to an SRT based on its WCET is often considered a waste of resources. At the price of an occasional deadline miss, SRT tasks are allocated less bandwidth than the worst case.

A task may access shared resources using mutex semaphores. If task $\tau_{i}$ accesses a resource $R_{k}$, we say that $\tau_{i}$ uses $R_{k}$. Without loss of generality, in the remainder of this paper, we will denote the resource and the corresponding mutex semaphore with the same symbol $R_{k}$. A critical section on resource $R_{k}$ is a section of code delimited by wait and signal operations on the corresponding semaphore, denoted by $P\left(R_{k}\right)$ and $V\left(R_{k}\right)$, respectively. Critical sections can be nested, i.e., it is possible to access resource $R_{j}$ while holding the lock on resource $R_{k}$. We assume properly nested critical sections, i.e., a sequence of code like $P\left(R_{1}\right), \ldots, P\left(R_{2}\right), \ldots, V\left(R_{2}\right), \ldots, V\left(R_{1}\right)$ is valid, whereas a sequence like $P\left(R_{1}\right), \ldots, P\left(R_{2}\right), \ldots$, $V\left(R_{1}\right), \ldots, V\left(R_{2}\right)$ is not valid. Internal critical sections are nested inside other critical sections, whereas external critical sections are not. We denote the worst-case execution time of the longest critical section of task $\tau_{i}$ on resource $R_{k}$ as $\xi_{i}\left(R_{k}\right)$. Note that $\xi_{i}\left(R_{k}\right)$ comprises the execution time of all the nested critical sections. We also assume that, if a job performs a wait operation on semaphore $R_{k}$, it performs the corresponding signal operation before its completion.

In this paper, we propose to schedule each task through a server. A server is an abstraction used by the scheduler to store the scheduling parameters of a task. It is characterized by a priority and it can be inserted in the system ready queue. Each task is associated with a server and, when the server is selected by the scheduler, the associated task is dispatched. Therefore, the server can be seen as a wrapper for a task. For the sake of simplicity, in the remainder of the paper, we will write "server $S_{i}$ is blocked," meaning that the task served by $S_{i}$ is blocked, and "server $S_{i}$ is executing," meaning that the task served by $S_{i}$ is executing. Each server is characterized by a budget $Q_{i}$ and a period $P_{i}$, with the interpretation being that each task is allowed to execute for $Q_{i}$ out of every $P_{i}$ time units. The fraction $U_{i}=\frac{Q_{i}}{P_{i}}$ represents the share (or bandwidth) of the processor reserved for server $S_{i}$. Our attention is restricted to systems in which all servers execute on a 
single shared processor and the sum of the processor shares of all the servers is no more than one:

$$
\left(\sum_{i=1}^{n} \frac{Q_{i}}{P_{i}}\right) \leq 1 .
$$

\subsection{Requirements}

In this paper, we present a novel scheduling policy and the schedulability analysis for HRT tasks. As a general rule, the scheduling algorithm should not be based on the knowledge of the temporal behavior of the tasks. However, it must allow a schedulability analysis. This separation of concerns between scheduling algorithm and schedulability analysis is very useful in dynamic real-time systems. Our goal is to find a scheduling algorithm that is able to provide the BIP without making any assumption on the temporal behavior of the tasks. If it is possible to exactly characterize a priori the temporal behavior of a task, then the proposed schedulability analysis can be used to compute the server's budget and period that guarantee the task's deadlines. However, if our analysis is not correct, the BIP guarantees that the other tasks in the system will not be affected.

Therefore, our scheduling algorithm must fulfill the following requirements:

- The arrival times of the jobs (the $a_{i, j} \mathrm{~s}$ ) are not known a priori, but are only revealed online during system execution. Hence, our scheduling strategy cannot require knowledge of future arrival times. For example, we do not require the tasks to be periodic.

- The exact execution requirements $c_{i, j}$ are also not known beforehand. They can only be determined by actually executing $J_{i, j}$ to completion. Nor do we require an a priori upper bound (a "worst-case execution time") on the value of $c_{i, j}$.

- The scheduling algorithm has no a priori knowledge of which resources a task will access; it can only be known online when the task tries to lock a resource. Nor do we require any a priori upper bound on the worst-case execution time $\xi_{i, j}$ of a critical section.

The last two assumptions are important because they rule out the use of protocols like the PCP. See Section 6.2 for a discussion on the PCP and the deadlock problem.

The following information is needed only for performing a schedulability analysis on an HRT task $\tau_{i}$ :

- $\quad$ the worst-case computation time $C_{i}$;

- $\quad$ the period $T_{i}$;

- the type (HRT or SRT) of every task that (directly or indirectly) interacts with $\tau_{i}$ (see Section 6.1 for a definition of interaction);

- for each interacting task $\tau_{j}$ and for each shared resource $R_{k}$, the worst-case execution time $\xi_{j}\left(R_{k}\right)$ of the longest critical section of $\tau_{j}$ on $R_{k}$.

\section{The Constant Bandwidth Server}

In this section, a brief overview of the Constant Bandwidth Server (CBS) algorithm is given. A fully detailed description can be found in [4]. A server $S_{i}$ is described by two parameters: the server maximum budget
$Q_{i}$, and the server period $P_{i}$. The server bandwidth $U_{i}=\frac{Q_{i}}{P_{i}}$ is the fraction of the CPU bandwidth assigned to $S_{i}$. The algorithm dynamically updates two variables $\left(q_{i}, \delta_{i}\right)$ for each server $S_{i}: q_{i}$ is the server's current budget and keeps track of the consumed bandwidth; $\delta_{i}$ is the server's current scheduling deadline. Initially, $q_{i}$ is set to the maximum budget $Q_{i}$ and $\delta_{i}$ is set to 0 . A server is active if the corresponding task has a pending instance.

All tasks in the system are assumed to be independent and no task is allowed to suspend itself waiting for a shared resource or a synchronisation event. The system consists of $n$ servers and a global scheduler based on the Earliest Deadline First (EDF) priority assignment. At each instant, the active server with the earliest scheduling deadline $\delta_{i}$ is selected and the corresponding task is dispatched to execute.

The CBS algorithm updates its variables as follows:

- Rule A: When job $J_{i, j}$ of task $\tau_{i}$ arrives at time $a_{i, j}$, the server checks the following condition:

$$
q_{i} \leq Q_{i} \frac{\delta_{i}-a_{i, j}}{P_{i}} .
$$

If the condition is verified, the current pair $\left(q_{i}, \delta_{i}\right)$ is used. Otherwise, a new pair $\left(q_{i}, \delta_{i}\right)$ is computed as $q_{i} \leftarrow Q_{i}$ and $\delta_{i} \leftarrow a_{i, j}+P_{i}$.

- Rule B: If server $S_{i}$ executes for $\Delta t$ units of time, the budget is decreased accordingly: $q_{i} \leftarrow q_{i}-\Delta t$.

- Rule C: Server $S_{i}$ is allowed to execute while $q_{i}>0$. When the budget is exhausted $\left(q_{i}=0\right)$ and the served job has not finished yet, a new pair $\left(q_{i}, \delta_{i}\right)$ is computed: The scheduling deadline is postponed to $\delta_{i} \leftarrow \delta_{i}+P_{i}$ and the budget is recharged to $q_{i} \leftarrow Q_{i}$. Since the scheduling deadline has changed, the EDF queue may be reordered and a preemption may occur.

If Rule C is never applied to server $S_{i}$ (i.e., it is never the case that $q_{i}=0$ and the task has not yet finished), we say that server $S_{i}$ never postpones its scheduling deadline.

The CBS algorithm has two important properties: the bandwidth isolation property (BIP) and the hard schedulability property (HSP). The BIP ensures that each server $S_{i}$ will contribute to the total system utilization for no more than $U_{i}$. The HSP ensures that it is possible to independently verify the schedulability of each HRT task. For proofs of the following theorems, please refer to [20] and [10].

Theorem 1 (Bandwidth Isolation Property). Given a system of $n$ servers, with $\sum_{i=1}^{n} U_{i} \leq 1$, no server misses its scheduling deadline, regardless of the behavior of the tasks.

Theorem 2 (Hard Schedulability Property). If an HRT task $\tau_{i}$ is served by a server $S_{i}=\left(Q_{i}, P_{i}\right)$, with maximum budget $Q_{i} \geq C_{i}$ and period $P_{i} \leq T_{i}$, then server $S_{i}$ never postpones its scheduling deadline and each job will complete before its absolute deadline.

The underlying assumption used to prove the previous theorems is that the executing server is the one with the earliest deadline. If a task is blocked on a semaphore, then this assumption is violated and the previous properties do not hold anymore. 


\section{The Priority Inheritance Protocol}

The Priority Inheritance Protocol (PIP) was first presented in [5] to solve the priority inversion problem. According to this protocol, when a high-priority job $J_{H}$ wants to access a critical section that is already held by a low-priority job $J_{L}$, the latter inherits the priority of $J_{H}$. When $J_{L}$ unlocks the resource, it returns to the priority it had at the time it acquired the lock.

Even though the PIP was developed in the context of fixed priority scheduling, it can be applied in the context of dynamic priority scheduling as well. The following basic properties hold:

- A job $J_{H}$ can be blocked by a lower priority job $J_{L}$ for at most the worst-case execution time of one critical section of $J_{L}$, regardless of the number of semaphores shared by $J_{H}$ and $J_{L}$.

- A job $J_{i}$ can encounter blocking by at most one critical section for each semaphore that it tries to lock.

Using these properties, it is possible to give a sufficient condition for the schedulability of a set of $n$ HRT periodic tasks. Suppose that the tasks are ordered by nondecreasing periods $\left(T_{i}<T_{j} \Rightarrow i<j\right)$. The schedulability condition is the following [21], [22]:

$$
\forall i \quad 1 \leq i \leq n \quad \sum_{j=1}^{i} \frac{C_{j}}{T_{j}}+\frac{B_{i}}{T_{i}} \leq 1,
$$

where $B_{i}$ is the worst-case blocking time of task $\tau_{i}$.

\subsection{Using the Priority Inheritance Protocol with the CBS}

When applying the PIP to the CBS, it is not clear how to account for the blocking time. One possible way would be to consider the blocking time using the following admission test:

$$
\forall i \quad 1 \leq i \leq n \quad \sum_{j=1}^{i} \frac{Q_{j}}{P_{j}}+\frac{B_{i}}{P_{i}} \leq 1,
$$

where $B_{i}$ represents the maximum blocking time experienced by each server.

However, this solution is not suitable for a dynamic system. In fact, to compute the maximum blocking time of each server, when a task is created we must "declare" the worst-case execution time of the critical sections on each accessed resource. This is in contrast with the goal of a scheduler that must be independent of the actual requirements of the tasks. In addition, if an SRT task holds a critical section for longer than declared, any server can miss its deadline.

Example 1. To highlight this problem, consider the example shown in Fig. 1. In this example, there are three servers, $S_{1}=(2,6), S_{2}=(2,6)$ and $S_{3}=(6,18)$. Server $S_{1}$ is assigned task $\tau_{1}$, which accesses a resource $R$ for the entire duration of its jobs (i.e., two units of time). Server $S_{2}$ is assigned task $\tau_{2}$, which does not use any resource. Server $S_{3}$ is assigned task $\tau_{3}$, which has an execution time of six units of time and accesses resource $R$ for five units of time. Now, suppose that $\tau_{3}$ is an SRT

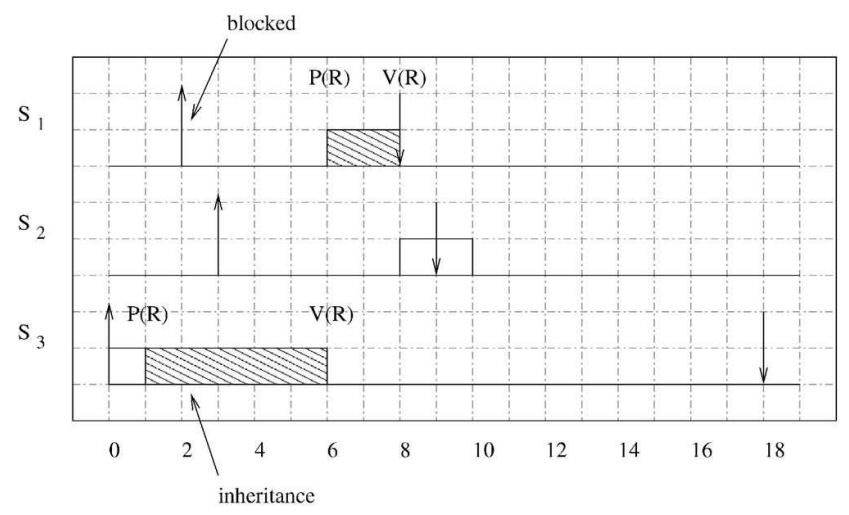

Fig. 1. In this example, blocking times are not correctly accounted for.

task that claims to use resource $R$ for only two units of time. The system computes a maximum blocking time $B_{1}=B_{2}=2$ for servers $S_{1}$ and $S_{2}$. According to (3), the system is schedulable and all servers can be admitted.

In the configuration of arrival times shown in Fig. 1, server $S_{1}$ arrives at time $t_{2}$ and tries to access $R$. Since it is locked, server $S_{3}$ inherits a deadline $\delta_{3}^{\prime}=8$ and continues executing. If no enforcement is put on the worst-case execution time of the critical section of task $\tau_{3}$ on resource $R$, server $S_{2}$ misses its deadline, as is shown in Fig. 1. The simple fact that $\tau_{3}$ executes more than expected inside the critical section invalidates the BIP and task $\tau_{2}$, which does not use any resource, misses its deadline.

Another problem that must be considered is the depletion of the server budget while the task is in a critical section and has inherited the deadline of another server. In the original CBS formulation, the server deadline is postponed and the server budget is immediately recharged. When the PIP is applied, it is not clear which deadline has to be postponed.

To solve the problems mentioned above, we combine the PIP and the CBS in a single protocol called BandWidth Inheritance (BWI). The basic idea is that, when a task that executes inside a low-priority server blocks a high-priority server, it inherits the pair $(q, \delta)$ of the blocked server.

\section{The Bandwidth Inheritance Protocol}

Before starting with the description of the Bandwidth Inheritance protocol, we need to understand the meaning of temporal isolation when considering interacting tasks. In the original CBS paper [4], tasks are assumed to be independent and, hence, do not interact in any way. When tasks access shared resources, they cannot be considered completely independent anymore. What does isolation mean in such a scenario?

Consider again the example shown in Fig. 1. Server $S_{1}$ and server $S_{3}$ share a resource. It is easy to see that if $S_{3}$ holds the lock for longer than declared, some task will probably miss its deadline. Our goal is to prevent task $\tau_{1}$ and $\tau_{3}$ from interfering with $\tau_{2}$. In fact, since $\tau_{1}$ and $\tau_{3}$ both access the same resource, it is impossible to provide isolation among them. 


\subsection{Bandwidth Isolation in the Presence of Shared Resources}

In this section, we define more precisely the concept of interaction between tasks. Intuitively, a task $\tau_{i}$ can be affected by a task $\tau_{j}$ if it can be directly or indirectly blocked by $\tau_{j}$. This relation is formalized by the following definition:

Definition 1. A sequence $H_{i}=\left(\tau_{1}, R_{1}, \tau_{2}, R_{2}, \ldots, R_{z-1}, \tau_{z}\right)$, with $z \geq 2$, is a blocking chain on task $\tau_{i}$ if:

- $\tau_{i}=\tau_{1}$

- $\forall k=1, \ldots, z-1, \tau_{k}$ and $\tau_{k+1}$ both use $R_{k}$; and

- If $z>2 \forall k=2, \ldots, z-1, \tau_{k}$ accesses $R_{k}$ with a critical section that is nested inside a critical section on $R_{k-1}$.

If $z=2$, then $\tau_{i}$ and $\tau_{z}$ directly share a resource. If $z>2$, then $\tau_{i}$ and $\tau_{z}$ interact through nested critical sections.

As an example, consider the blocking chain $H_{1}=\left(\tau_{1}, R_{1}, \tau_{2}, R_{2}, \tau_{3}\right)$ :

- $\quad$ Task $\tau_{3}$ uses resource $R_{2}$;

- Task $\tau_{2}$ uses $R_{2}$ with a critical section that is nested inside the critical section on $R_{1}$; and

- $\quad$ Task $\tau_{1}$ uses $R_{1}$.

Notice that, in the above example, $\tau_{1}$ can be blocked by $\tau_{2}$ and by $\tau_{3}$, but $\tau_{3}$ cannot be blocked by $\tau_{1}$. Hence, a blocking chain defines an antisymmetric relation $\models$ between $\tau_{i}$ and $\tau_{z}: \tau_{i} \models \tau_{z}$ but not vice versa.

In general, there can be more than one chain between two tasks $\tau_{i}$ and $\tau_{j}$ because they can directly or indirectly share more than one resource. Let us enumerate the chains starting from task $\tau_{i}$ in any order. $H_{i}^{h}$ denote the $h$ th blocking chain on $\tau_{i}$. Without loss of generality, in the remainder of the paper, we will sometimes drop the superscript on the chain.

Let $\Gamma\left(H_{i}\right)$ denote the set of tasks $\tau_{2}, \ldots, \tau_{z}$ in the sequence $H_{i}$ ( $\tau_{i}$ excluded), and $R\left(H_{i}\right)$ the set of resources $R_{1}, \ldots, R_{z-1}$ in the sequence $H_{i}$, respectively.

Definition 2. The set $\Gamma_{i}$ of tasks that may interact with $\tau_{i}$ is defined as follows:

$$
\Gamma_{i}=\bigcup_{h} \Gamma\left(H_{i}^{h}\right) .
$$

Set $\Gamma_{i}$ comprises all tasks that may directly or indirectly block $\tau_{i}$.

Given these definitions, we can state more precisely the goals of our scheduling strategy. Whether task $\tau_{i}$ meets its deadlines should depend only on the timing requirements of $\tau_{i}$ and on the worst-case execution time of the critical sections of the tasks in $\Gamma_{i}$. Therefore, in order to guarantee an HRT task $\tau_{i}$, it is only necessary to know the behavior of the tasks in $\Gamma_{i}$.

\subsection{The Priority Ceiling Protocol and the Problem of Deadlock}

If we allow nested critical section, the problem of deadlock must be taken into account. Deadlock can be avoided by means of static or dynamic policies. One possibility is to use a protocol, like the $\mathrm{PCP}$, that prevents deadlock from occurring.
However, the PCP uses a priori information on the task parameters. For each resource, it requires the knowledge of the priorities of all the accessing tasks in order to compute the resource ceiling. The resource ceilings are then used by the protocol to regulate access to resources. Therefore, the requirements of the PCP are in contrast with the initial requirements we made for our scheduling discipline. In addition, if the ceilings are not computed correctly, not only the analysis but also the resulting schedule is incorrect. An example can be easily built in which, due to an SRT task that fails to declare access to one resource, the ceilings are not computed correctly and a deadlock can occur.

On the other hand, the PIP does not require any a priori information. Hence, in this paper, we select the PIP as the basic resource access protocol.

To solve the deadlock problem, we consider another static policy. We assume that resources are totally ordered and each task respects the ordering in accessing nested critical sections. Thus, if $i<j$, then task $\tau$ can access a resource $R_{j}$ with a critical section that is nested inside another critical section on resource $R_{i}$. When such an order is defined, the sequence of resources in any blocking chain is naturally ordered. For a deadlock to be possible, a blocking chain must exist in which there is a circular relationship like $H=\left(\ldots, R_{i}, \ldots, R_{j}, \ldots R_{i}, \ldots\right)$. Therefore, if the resources are ordered a priori, a deadlock cannot occur.

If the total order is not respected when accessing nested critical sections, a deadlock can still occur. As we will see in the next section, our scheduler is able to detect it during runtime, but the action to be taken depends on the kind of resources. In the remainder of the paper, we shall assume that resources are ordered.

\subsection{Description of the Bandwidth Inheritance Protocol}

The BWI protocol allows tasks to be executed on more than one server. Every server $S_{i}$ maintains a list of served tasks. During runtime, it can happen that a task $\tau_{i}$ is in the list of more than one server. Let $e(i, t)$ be the index of the earliest deadline server among all the servers that have $\tau_{i}$ in their list at time $t$. Initially, each server $S_{i}$ has only its own task $\tau_{i}$ in the list, hence, $\forall i e(i, 0)=i{ }^{1}$ We call server $S_{i}$ the default server for task $\tau_{i}$.

As long as no task is blocked, BWI follows the same rules as Algorithm CBS. In addition, BWI introduces the following rules:

- Rule D: If task $\tau_{i}$ is blocked when accessing a resource $R$ that is locked by task $\tau_{j}$, then $\tau_{j}$ is added to the list of server $S_{e(i, t)}$. If, in turn, $\tau_{j}$ is currently blocked on some other resource, then the chain of blocked tasks is followed and server $S_{e(i, t)}$ adds all the tasks in the chain to its list until it finds a ready task. $^{2}$ In this way, each server can have more than one task to serve, but only one of these tasks is not blocked.

1. Note that index $i$ denotes the task's index when it is the argument of function $e()$ and the server's index when it is the value of $e()$.

2. If, by following the chain, the protocol finds a task that is already in the list, a deadlock is detected and an exception is raised. 


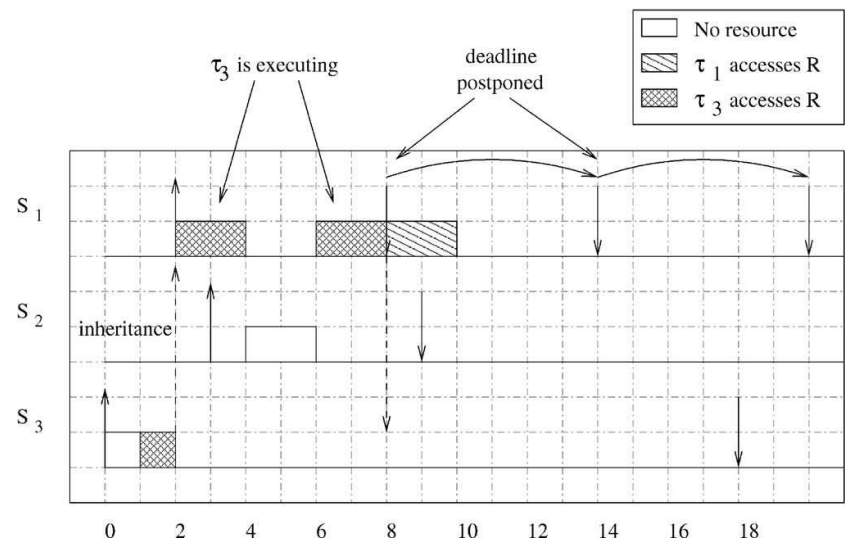

Fig. 2. BWI is applied to the example of Fig. 1.

- Rule E: When task $\tau_{j}$ releases resource $R$, if there is any task blocked on $R$, then $\tau_{j}$ was executing inside a server $S_{e(j, t)} \neq S_{j}$. Server $S_{e(j, t)}$ must now discard $\tau_{j}$ from its own list and the first blocked task in the list is now unblocked, let it be $\tau_{i}$. All the servers that added $\tau_{j}$ to their list while $\tau_{j}$ was holding $R$ must discard $\tau_{j}$ and add $\tau_{i}$.

BWI is an inheritance protocol: When a high-priority task $\tau_{i}$ is blocked by a lower-priority task $\tau_{j}, \tau_{j}$ inherits server $S_{e(i, t)}$ and the execution time of $\tau_{j}$ is then charged to $S_{e(i, t)}$. Therefore, $S_{e(j, t)}=S_{e(i, t)}$. When the budget of $S_{e(i, t)}$ is exhausted, $S_{e(i, t)}$ 's deadline is postponed and $\tau_{j}$ can continue to execute on server $S_{e(j, t)}$ that may now be different from $S_{e(i, t)}$.

Example 2. The behavior of BWI is demonstrated by applying the protocol to the example of Fig. 1. The resulting schedule is depicted in Fig. 2.

- At time $t=1$, task $\tau_{3}$, which is initially served by $S_{3}$, locks resource $R$.

- At time $t=2$, server $S_{1}$ becomes the earliest deadline server and dispatches task $\tau_{1}$, which immediately tries to lock resource $R$. However, the resource is already locked by $\tau_{3}$. According to Rule D, $\tau_{3}$ is added to the list of $S_{1}$ and $\tau_{1}$ is blocked. Now, there are two task in $S_{1}$ 's list, but only $\tau_{3}$ is ready. Hence, $S_{e(3,2)}=S_{1}$ and $S_{1}$ dispatches task $\tau_{3}$.

- At time $t=3$, server $S_{2}$ is activated, but it is not the earliest deadline server. Thus, $\tau_{3}$ continues to execute.

- At time $t=4$, the budget of server $S_{1}$ has been depleted. According to Rule C (see Section 4), the server deadline is postponed to $\delta_{1} \leftarrow \delta_{1}+P_{1}=14$ and the budget is recharged to $q_{1} \leftarrow Q_{1}=2$. Since $S_{1}$ is no longer the earliest deadline server, $S_{2}$ is selected and task $\tau_{2}$ is dispatched.

- At time $t=6, S_{1}$ is again the earliest deadline server; hence, task $\tau_{3}$ is dispatched.

- At time $t=8, \tau_{3}$ releases the lock on $R$. According to Rule E, $\tau_{1}$ is unblocked and $\tau_{3}$ is discarded from the list of server $S_{1}$. Now, $S_{1}$ 's list contains only task $\tau_{1}$ and $S_{e(3,2)}=S_{3}$. Server $S_{1}$ is still the earliest deadline server, but its budget has been depleted. According to Rule C, $\delta_{1} \leftarrow \delta_{1}+P_{1}=20$ and $q_{1} \leftarrow Q_{1}=2$.

Note that, in this case, task $\tau_{2}$ is not influenced by the misbehavior of $\tau_{3}$ and completes before its deadline.

\subsection{Properties of BWI}

In this section, the bandwidth isolation property and the hard schedulability property are extended to consider interacting tasks and they are proven for the BWI protocol. We also derive sufficient conditions for assigning server parameters that guarantee HRT tasks. First, we present some preliminary results.

Lemma 1. Each active server always has exactly one ready task in its list.

Proof. Initially, no task is blocked and the lemma is true. Suppose that the lemma holds just before time $t_{b}$, when task $\tau_{i}$ is blocked on resource $R$ by task $\tau_{j}$. After applying Rule D, both servers $S_{j}$ and $S_{i}$ have task $\tau_{j}$ in their list and task $\tau_{i}$ is blocked. By definition of $e\left(j, t_{b}\right), S_{e\left(j, t_{b}\right)}=S_{i}$. Moreover, if $\tau_{j}$ is also blocked on another resource, the blocking chain is followed and all the blocked tasks are added to $S_{i}$ until the first nonblocked task is reached. The lists of all the other servers remain unchanged, thus the lemma is true.

Now, suppose that the lemma is true just before time $t_{r}$. At this time, task $\tau_{j}$ releases the lock on resource $R$. If no other task was blocked on $R$, then the lists of all the servers remain unchanged. Otherwise, suppose that task $\tau_{i}$ was blocked on $R$ and is now unblocked: Server $S_{i}$ has $\tau_{j}$ and $\tau_{i}$ in its list and, by applying Rule E, discards $\tau_{j}$. The lists of all the other servers remain unchanged, and the lemma holds.

Theorem 3. Consider a system consisting of $n$ servers with $\sum_{i=1}^{n} U_{i} \leq 1$, which uses the BWI protocol for accessing shared resources. No server in the system misses its scheduling deadline.

Proof. Lemma 1 implies that, at all times, the earliest deadline server has one and only one ready task in its list. As explained in [10], the resulting schedule can be regarded, from the viewpoint of the global scheduler, as a sequence of real-time jobs whose deadlines are equal to the deadlines of the servers (also referred as chunks in [20] and [4]). As the earliest deadline server never blocks, the computation times and the deadlines of the chunks generated by the server do not depend on the presence of shared resources. In [20], [10], it was proven that, in every interval of time, the bandwidth demanded by the chunks produced by server $S_{i}$ never exceeds $\frac{Q_{i}}{P_{i}}$, regardless of the behavior of the served tasks. Since Lemma 1 states that each active server always has one nonblocked task in its list, the previous result is also valid for BWI. Hence, from the optimality of EDF and from $\sum_{i=1}^{n} \frac{Q_{i}}{P_{i}} \leq 1$, it follows that none of these chunks misses its deadline.

Note that the previous theorem states that no scheduling deadline (that is, we recall, the server's deadline used to schedule the servers with EDF) will be missed, but it does 
not say anything about a task's deadlines. Recall that the goal of a real-time scheduling algorithm is to meet the HRT deadlines. At this point, we need a way to relate the tasks' deadlines to the server deadlines (and, hence, to the server parameters) so that it is possible to provide guarantees to HRT tasks.

Definition 3. Given a task $\tau_{i}$, served by a server $S_{i}$ with the BWI protocol, the interference time $I_{i}$ is defined as the maximum time that all other tasks can execute inside server $S_{i}$ for each job of $\tau_{i}$.

Theorem 4. If an HRT task $\tau_{i}$ is served by a server $S_{i}$ with the BWI protocol, with parameters $Q_{i}=C_{i}+I_{i}$ and $P_{i}=T_{i}$, where $C_{i}$ is the WCET, $T_{i}$ is the minimum interarrival time, and $I_{i}$ is the maximum interference time for $S_{i}$, then task $\tau_{i}$ will meet all its deadlines, regardless of the behavior of the other noninteracting tasks in the system.

Proof. According to Theorem 2, the CBS algorithm guarantees that each server $S_{i}$ receives up to $Q_{i}$ units of execution every $P_{i}$ units of time. Hence, if each instance of $\tau_{i}$ consumes less than $Q_{i}$ and instances are separated by $P_{i}$ or more, server $S_{i}$ never postpones its scheduling deadline. From Theorem $1, f_{i, j} \leq \delta_{i}$.

Theorem 3 extends the result of Theorem 2 to BWI. However, when considering the BWI protocol, other tasks can execute inside server $S_{i}$, consuming its budget (and, hence, postponing the deadline of server $S_{i}$ even if $C_{i} \leq Q_{i}$ ). In order to ensure that server $S_{i}$ will never postpone its scheduling deadline, we have to consider the interference time from those tasks. If $I_{i}$ is the maximum time that other tasks can execute inside $S_{i}$, it follows that $\tau_{i}$ can execute for $Q_{i}-I_{i}$ units of time before exhausting the server budget. Hence, the theorem follows.

\subsubsection{Considerations}

When our system consists only of HRT tasks, BWI is not the best protocol to use. In fact, substituting $Q_{i}$ and $P_{i}$ into (1), we obtain:

$$
\sum_{i=1}^{n} \frac{C_{i}+I_{i}}{T_{i}} \leq 1
$$

which may result in a lower utilization than (2) because all the interference times are summed together. Hence, if we are dealing with a hard real-time system, it is better to use other scheduling strategies like the PCP [5] or the SRP [17], [2].

The BWI protocol is more suitable for dynamic real-time systems, where hard, soft, and non-real-time tasks can coexist and it is impossible to perform an offline analysis for the entire system. Of course, this comes at the cost of a lower utilization for HRT tasks.

\section{INTERFERENCE TIME Computation}

In the general case, an exact computation of $I_{i}$ is a complex problem. In this section, we restrict our attention to the computation of the interference time for HRT tasks. At first glance, the problem may seem similar to the problem of computing the blocking times $B_{i}$ for the PIP. However, computing the interference time is much more difficult because we have to consider the interference of the SRT tasks. Their unpredictable execution times may cause the associated servers to exhaust their budgets and postpone their deadlines.

In many cases, it is desirable to guarantee an HRT task $\tau_{i}$ even if it interacts with SRT tasks. In fact, sometimes it is possible to know indirectly the worst-case execution time of the critical sections of an SRT task. For example, consider an HRT task and an SRT task that access the same resource by using common library functions. If the critical sections are implemented as library functions with bounded execution time, then we can still determine the amount of time that a soft task can steal from the server's budget of an HRT task. Indeed, this is a very common case in a real operating system.

Therefore, we will now consider the problem of computing $I_{i}$ for a server $S_{i}$ that is the default server of an HRT task. We start by providing an important definition that simplifies the discussion.

Definition 4. Let $S_{i}$ be a server that never postpones its deadline (i.e., $S_{i}$ 's budget is never exhausted while there is a job that has not yet finished). We call $S_{i}$ an HRT server. If the server deadline of $S_{i}$ can be postponed (i.e., a time $t$ exists in which $q_{i}=0$ and the served job has not yet finished), we call $S_{i}$ an SRT server.

The distinction between HRT and SRT servers depends only on the kind of tasks they serve. Both HRT and SRT servers follow the same rules and have the same characteristics. However, it may be impossible to know the WCET of an SRT task, so the corresponding default SRT server can decrease its priority while executing. The presence of SRT servers that interact with HRT servers complicates the computation of the interference time.

The following examples show how one or more SRT tasks can contribute (directly or indirectly) to the interference time of an HRT task.

Example 3. Consider an HRT task $\tau_{i}$, served by server $S_{i}$ and an SRT task $\tau_{j}$, served by a server $S_{j}$ with period $P_{j}<P_{i}$. We do not know the WCET of task $\tau_{j}$. Therefore, we assign the budget of $S_{j}$ according to some rule of thumb. Server $S_{j}$ is an SRT server as it may postpone its deadline. If $\tau_{j}$ executes less than its server budget and the server deadline is not postponed, $S_{i}$ cannot preempt $S_{j}$. If, instead, $\tau_{j}$ executes for more than its server budget, the server's deadline is postponed. The corresponding situation is shown in Fig. 3a. $S_{j}$ can be preempted by $S_{i}$ while inside a critical section, and block $\tau_{i}$, contributing to its interference time $I_{i}$.

Example 4. Consider three tasks, $\tau_{i}, \tau_{j}$, and $\tau_{k}$, served by servers $S_{i}, S_{j}$, and $S_{k}$, respectively, with $P_{j}<P_{i}<P_{k}$. Servers $S_{i}$ and $S_{k}$ are HRT servers, while $S_{j}$ is an SRT server. All tasks access resource $R$. Task $\tau_{i}$ accesses resource $R$ twice with two different critical sections. One possible blocking situation is shown in Fig. 3b. The first time, $\tau_{i}$ can be blocked by task $\tau_{k}$ on the first critical section. Then, it can be preempted by task $\tau_{j}$, which first locks $R$, and then, before releasing the resource, depletes the server budget and postpones its deadline. Thus, 


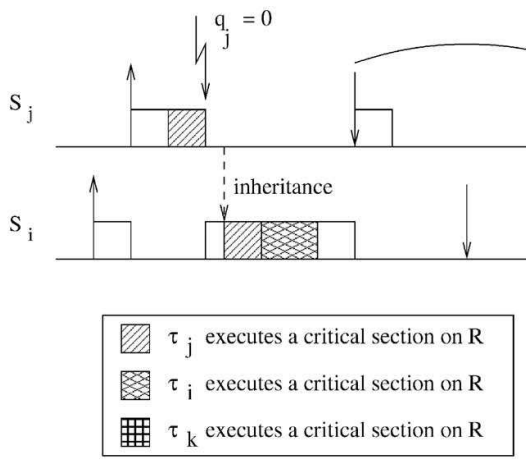

(a)

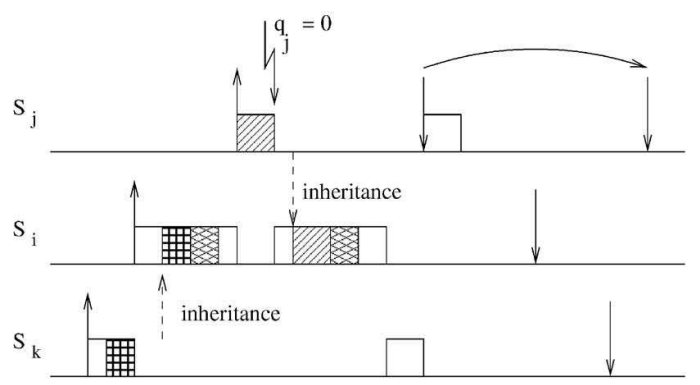

(b)

Fig. 3. Example of blocking situations with SRT tasks: (a) An SRT task with a short period blocks an HRT task with a long period. (b) An HRT task is blocked twice on resource $\mathrm{R}$.

when $\tau_{i}$ executes, it can be blocked again on the second critical section on $R$. Note that both $\tau_{j}$ and $\tau_{k}$ belong to $\Gamma_{i}$.

Example 5. As the last example, we show one case in which, even if all tasks in $\Gamma_{i}$ are HRT tasks, it may happen that $\tau_{j}$ interferes with $S_{i}$ with two different critical sections. Consider three tasks, $\tau_{i}, \tau_{j}$, and $\tau_{k}$. Task $\tau_{i}$ accesses only resource $R_{2}$ with two critical sections. Task $\tau_{j}$ accesses two resources $R_{1}$ and $R_{2}$ and $R_{2}$ is accessed twice with two critical sections, both nested inside the critical section on $R_{1}$. Task $\tau_{k}$ accesses only $R_{1}$ with one critical section. The only blocking chain starting from task $\tau_{i}$ is $H_{i}=\left(\tau_{i}, R_{2}, \tau_{j}\right)$. Hence, $\Gamma_{i}=\left\{\tau_{j}\right\}$. Note that task $\tau_{k}$ cannot interfere with task $\tau_{i}$.

Tasks $\tau_{i}, \tau_{j}$, and $\tau_{k}$ are assigned servers $S_{i}, S_{j}$, and $S_{k}$, respectively, with $P_{k}<P_{i}<P_{j}$. Tasks $\tau_{i}$ and $\tau_{j}$ are both HRT tasks and we know their WCETs and periods. Task $\tau_{k}$ is an SRT task and we do not know its WCET. Finally, we assume knowing the duration of all critical sections (for example, because resources are accessed through shared libraries that we are able to analyze).

We assign budgets and periods of servers $S_{i}$ and $S_{j}$ so that they are HRT servers (to compute their interference time, we use the algorithm described in Fig. 5, which will be presented later). We assign the budget of server $S_{k}$ according to some rule of thumb. We do not know if $S_{k}$ will exhaust its budget while executing. Therefore, we consider server $S_{k}$ as an SRT server.
One possible blocking situation is shown in Fig. 4. Task $\tau_{j}$ locks resource $R_{1}$ and then resource $R_{2}$. At time $t_{1}$, it is preempted by task $\tau_{i}$ that tries to lock resource $R_{2}$ and it is blocked. As a consequence, task $\tau_{j}$ inherits server $S_{i}$ and interferes with it for the duration of the first critical section on $R_{2}$. When $\tau_{j}$ releases $R_{2}$, it returns inside its server $S_{j}$ and $\tau_{i}$ executes completing its critical section on $R_{2}$. Then, server $S_{k}$ is activated and $\tau_{k}$ starts executing and tries to lock resource $R_{1}$. Since $R_{1}$ is still locked by $\tau_{j}, \tau_{k}$ is blocked and $\tau_{j}$ inherits server $S_{k}$. While $\tau_{j}$ executes inside $S_{k}$, it again locks resource $R_{2}$. Before releasing $R_{2}$, server $S_{k}$ exhausts its budget and postpones its deadline. Now, the earliest deadline server is $S_{i}$ that continues to execute and tries to again lock $R_{2}$ at time $t_{2}$. As a consequence, $\tau_{j}$ inherits $S_{i}$ and interferes with it for the second time.

From the examples shown above, it is clear that there are many possible situations in which a task can interfere with a server. In the next section, we formally present a set of lemmas that identify the conditions under which a task can interfere with an HRT server.

\subsection{Conditions for Interference}

We start by defining the set of servers that can be inherited by a task.

Definition 5. Set $\Psi_{j}$ is the set of all servers that can be "inherited" by task $\tau_{j}, S_{j}$ included:

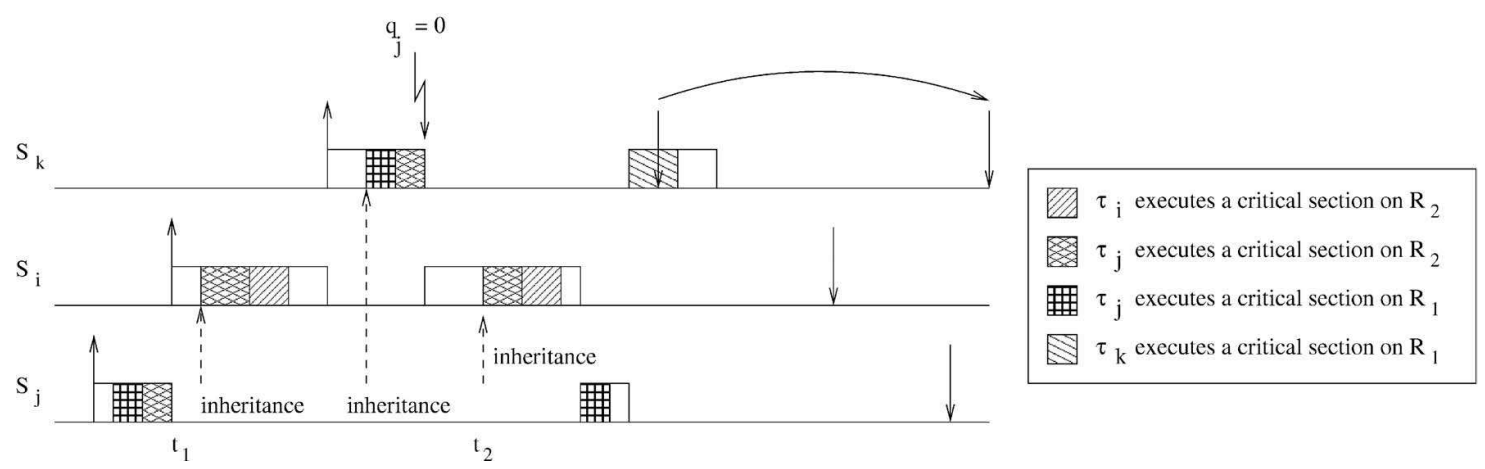

Fig. 4. Example of blocking situation: Task $\tau_{j}$ can interfere twice with $S_{i}$ even if $S_{i}$ and $S_{j}$ are both HRT servers. 


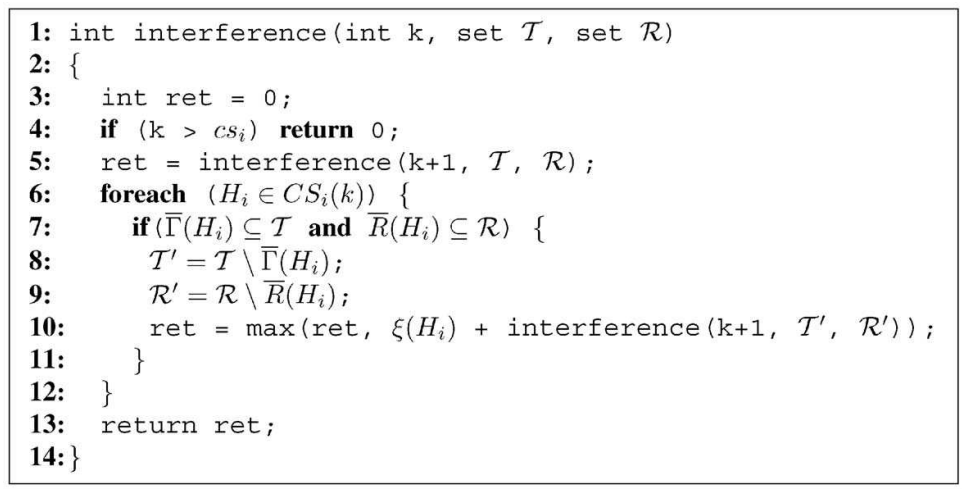

Fig. 5. Algorithm for computing the interference time for server $S_{i}$.

$$
\Psi_{j}=\left\{S_{i} \mid \exists H_{i}, \quad \tau_{j} \in H_{i}\right\} \cup\left\{S_{j}\right\} .
$$

A task $\tau_{j}$ can only inherit tasks in $\Psi_{j}$, hence $\forall t S_{e(j, t)} \in \Psi_{j}$.

Definition 6. Set $\Psi_{j}^{S R T}(i)$ is the set of SRT servers that can be "inherited" by task $\tau_{j}$ and interfere with server $S_{i}$ :

$$
\begin{gathered}
\Psi_{j}^{S R T}(i)=\left\{S_{k} \mid S_{k}\right. \text { is an SRT server^ } \\
\left.\exists H_{k}=\left(\tau_{k}, \ldots, \tau_{j}, \ldots, \tau_{i}\right)\right\} .
\end{gathered}
$$

If $S_{j}$ is an SRT server, it is also included in $\Psi_{j}^{S R T}(i)$.

Consider again Example 5. There is one chain from $\tau_{k}$ to $\tau_{i}: H_{k}=\left(\tau_{k}, R_{1}, \tau_{j}, R_{2}, \tau_{i}\right)$. Therefore, $S_{k} \in \Psi_{j}^{S R T}(i)$. Set $\Psi_{j}^{S R T}(i)$ is important in our analysis because it identifies whether a task $\tau_{j}$ can inherit an SRT server before interfering with the server $S_{i}$ under analysis. In Example 5, task $\tau_{j}$ can inherit the SRT server $S_{k}$, which may later postpone its deadline.

Now, we prove some important properties of the BWI protocol.

Lemma 2. If server $S_{i}$ is HRT, then $\forall t: \delta_{e(i, t)} \leq \delta_{i}$.

Proof. When $\tau_{i}$ inherits a server $S_{j}$, this server must have a scheduling deadline shorter than $\delta_{i}$. Recall that, by definition, $e(i, t)$ is the index of the server with the shortest scheduling deadline among all servers inherited by $\tau_{i}$ at time $t$. Hence, $\delta_{e(i, t)}=\delta_{j}<\delta_{i}$. If $S_{j}$ postpones its deadline before the time in which $\tau_{i}$ releases the resource, $\tau_{i}$ continues to execute inside the server with the shortest deadline among the inherited servers. Since $S_{i}$ never postpones its deadline, the lemma is proven. $\square$

Lemma 3. Given a task $\tau_{i}$, only tasks in $\Gamma_{i}$ can be added to server $S_{i}$ and contribute to $I_{i}$.

Proof. It follows directly from Rule D and from the definition of $\Gamma_{i}$.

Lemma 4. Let $S_{i}$ be an HRT server. Task $\tau_{j}$ with default server $S_{j}$ cannot interfere with server $S_{i}$ if:

$$
P_{j} \leq P_{i} \quad \wedge \quad \forall S_{k} \in \Psi_{j}^{S R T}(i): P_{k}>P_{i} .
$$

Proof. By contradiction. For $\tau_{j}$ to interfere with $S_{i}$, it must happen that, at a certain time $t_{1}, \tau_{j}$ locks a resource $R$; it is then preempted by server $S_{i}$ at time $t_{2}$, which blocks on some resource; $\tau_{j}$ inherits $S_{i}$ as a consequence of this blocking. Therefore, $\tau_{j}$ must start executing inside its default server before $S_{i}$ arrives and executes in a server $S_{e\left(j, t_{2}\right)}$ with deadline $\delta_{e\left(j, t_{2}\right)}>\delta_{i}$ when it is preempted. By hypothesis, $P_{j} \leq P_{i} \Rightarrow \delta_{j}<\delta_{i}$. Hence, $\tau_{j}$ executes in a server $S_{e\left(j, t_{1}\right)}$ with $\delta_{e\left(j, t_{1}\right)}>\delta_{i}$. However, it follows from the hypothesis that server $S_{j}$ never postpones its deadline $\left(S_{j} \notin \Psi_{j}^{S R T}(i)\right)$ and, from Lemma $2, \delta_{e\left(j, t_{1}\right)} \leq \delta_{j}<\delta_{i}$. This is a contradiction, hence the lemma is proven.

Now, we are ready to define more precisely which tasks can interfere with our server $S_{i}$.

Definition 7. A proper blocking chain $H_{i}$ is a blocking chain that contains only tasks that can interfere with $S_{i}$ :

$$
\forall \tau_{j} \in H_{i}: P_{j}>P_{i} \quad \vee \quad \exists S_{k} \in \Psi_{j}^{S R T}(i): P_{k} \leq P_{i} .
$$

Later, we will present an algorithm for computing the interference time for server $S_{i}$ which explores all proper blocking chains starting from $\tau_{i}$. However, in some cases, we have to consider multiple interference times from the same task and multiple interference times on the same resources. The following lemmas restrict the number of possible interference situations.

Lemma 5. Let $S_{i}$ be an HRT server and $\tau_{j}$ a task belonging to a proper blocking chain $H_{i}$. If the following condition holds:

$$
P_{j}>P_{i} \wedge \quad \forall S_{k} \in \Psi_{j}^{S R T}(i): P_{k} \geq P_{i},
$$

then $\tau_{j}$ can interfere with server $S_{i}$ for at most the worst-case execution of one critical section for each job.

Proof. Suppose that $\tau_{j}$ interferes with $S_{i}$ in two different intervals. The first time it interferes in interval $\left[t_{1}, t_{2}\right)$, the second time in interval $\left[t_{3}, t_{4}\right)$. Therefore, at time $t_{2}$, $\delta_{e\left(j, t_{2}\right)}>\delta_{i}$. If $\tau_{j}$ does not lock any resource in $\left[t_{2}, t_{3}\right)$, then, at time $t_{3}$, server $S_{i}$ blocks on some resource $R$ that was locked by $\tau_{j}$ before $t_{1}$ and that it has not yet released. Therefore, $\tau_{j}$ interferes with $S_{i}$ for the duration of the critical section on $R$, which includes the duration of the first critical section $\left(\xi(R) \geq\left(t_{4}-t_{3}\right)+\left(t_{2}-t_{1}\right)\right)$ and the lemma is proven.

Now, suppose that $\tau_{j}$ executes in interval $\left[t_{2}, t_{3}\right)$ and locks another resource $R_{1}$. It follows that it inherits a server $S_{k}$ that preempts $S_{i}$ with $\delta_{k}<\delta_{i}$. Hence, $P_{k}<P_{i}$. From the hypothesis, $S_{k}$ is an HRT server and $\delta_{k}$ is not 
postponed before $\tau_{j}$ releases resource $R_{1}$. Hence, $\tau_{j}$ cannot inherit $S_{i}$ while it is inside $S_{k}$ and we fall back to the previous case.

Lemma 6. Let $S_{i}$ be an HRT server and $R$ a resource. If the following condition holds:

$$
\forall H_{i}^{h}, H_{i}^{h}=\left(\ldots, R, \tau_{j}, \ldots\right), \quad \forall S_{k} \in \Psi_{j}^{S R T}(i): P_{k} \geq P_{i},
$$

then at most one critical section on $R$ can contribute to interference time $I_{i}$.

Proof. The proof of this lemma is very similar to the proof of Lemma 5. By contradiction. Suppose that two critical sections on the same resource $R$ contribute to $I_{i}$. The first time, task $\tau_{p}$ inherits server $S_{i}$ at time $t_{1}$ while it is holding the lock on $R$. The second time, task $\tau_{j}$ inherits server $S_{i}$ at time $t_{2}>t_{1}$ while it is holding the lock on $R$. It follows that:

- $\quad$ The lock on $R$ was released between $t_{1}$ and $t_{2}$;

- $\tau_{j}$ arrives before $t_{2}$ and executes on a server $S_{e(j)}$ with $\delta_{e(j)}<\delta_{i}$;

- $\tau_{j}$ acquires the lock on $R$ at $t_{a}<t_{2}$;

- just before $t_{2}, \delta_{i}<\delta_{e(j), t_{2}}$.

Hence, at time $t_{a}, \tau_{j}$ is executing in an inherited server $S_{k} \in \Psi_{j}^{S R T}(i)$ that postpones its deadline before $\tau_{j}$ releases the lock on $R$. $S_{k}$ must arrive after $S_{i}$ with deadline $\delta_{k}<\delta_{i}$ and later postpone its deadline. This contradicts the hypothesis that $\forall S_{k} \in \Psi_{j}^{S R T}(i): P_{k} \geq P_{i}$. Hence, the lemma is proven.

The previous lemmas restrict the number of combinations that we must analyze when computing the interference time. In particular, Lemma 5 identifies the conditions under which a task can interfere with server $S_{i}$ for at most one critical section; Lemma 6 identifies the conditions under which a certain resource can interfere with server $S_{i}$ at most one time.

Now, we need to quantify the interference time due to each blocking chain.

Lemma 7. The worst-case interference time for server $S_{i}$ due to a proper blocking chain $H_{i}=\left(\tau_{1}, R_{1}, \ldots, R_{z-1}, \tau_{z}\right)$ is:

$$
\xi\left(H_{i}\right)=\sum_{k=2}^{z} \xi_{k}\left(R_{k-1}\right) .
$$

Proof. It simply follows from the definition of proper blocking chain.

Given a proper blocking chain, we need to distinguish the tasks that can interfere with $S_{i}$ for at most the duration of one critical section (i.e., that verify the hypothesis of Lemma 5) from the task that can interfere with $S_{i}$ multiple times.

Definition 8. Given a proper blocking chain $H_{i}^{h}$, let $\bar{\Gamma}\left(H_{i}^{h}\right)$ be the set of tasks in $H_{i}^{h}$ that verify the hypothesis of Lemma 5.

$\bar{\Gamma}\left(H_{i}^{h}\right)=\left\{\tau_{j} \mid \tau_{j} \in H_{i}^{h} \wedge P_{j}>P_{i} \wedge\left(\forall S_{k} \in \Psi_{j}^{S R T}(i): P_{k} \geq P_{i}\right)\right\}$.

We do the same thing for the resources.

Definition 9. Given a proper blocking chain $H_{i}^{h}$, let $\bar{R}\left(H_{i}^{h}\right)$ be the set of resources in $H_{i}^{h}$ that verify the hypothesis of Lemma 6.

$$
\begin{aligned}
& \bar{R}\left(H_{i}^{h}\right)= \\
& \left\{R_{j} \mid R_{j} \in H_{i}^{h} \wedge P_{j+1}>P_{i} \wedge\left(\forall S_{k} \in \Psi_{j+1}^{S} R T(i): P_{k} \geq P_{i}\right)\right\} .
\end{aligned}
$$

\subsection{Algorithm for Computing the Interference Time}

We are now ready to present the pseudocode for Algorithm interference(), shown in Fig. 5. Let $c s_{i}$ denote the number of critical sections for task $\tau_{i}$ and $C S_{i}(k)$ denote the set of proper blocking chains starting from the $k$ th critical. More formally, $C S_{i}(k)$ is the set of proper blocking chains of the form $H_{i}^{h}=\left(\tau_{i}, R, \ldots\right)$, where $R$ is the resource accessed in the $k$ th critical section of $\tau_{i}$.

Function interference $(\mathrm{k}, \mathcal{T}, \mathcal{R})$ is first called with $k=1$, $\mathcal{T}=\Gamma_{i}$, and with $\mathcal{R}$ equal to the set of all possible resources. At line 5, we consider the case in which $\tau_{i}$ is not blocked on the $k$ th critical section. In this case, the function is recursively called for the $(k+1)$ th critical section.

At lines 6-12, we consider the case in which $\tau_{i}$ is blocked on the $k$ th critical section. For each proper blocking chain $H_{i}$ in $C S_{i}(k)$, the algorithm checks if it is a legal blocking chain, i.e., the resources in $\bar{R}\left(H_{i}^{k}\right)$ and the tasks in $\bar{\Gamma}\left(H_{i}^{k}\right)$ have not yet been considered in the interference time computation. If so, function interference () is recursively called with $k^{\prime}=k+1, \mathcal{T}^{\prime}=\mathcal{T} \backslash \bar{\Gamma}\left(H_{i}\right)$, and $\mathcal{R}^{\prime}=\mathcal{R} \backslash \bar{R}\left(H_{i}\right)$ (lines 8-10). Otherwise, it selects another chain from $C S_{i}(k)$. The recursion stops when $k>c s_{i}$ (line 4 ).

The algorithm has exponential complexity since it explores all possible interference situations for server $S_{i}$. We conjecture that the problem of finding the interference time in the general case is NP-Hard. However, we leave the proof of this claim as future work.

\subsection{Interference Time Computation for SRT Tasks}

It is very difficult to compute the interference time for an SRT task. The problem is that an SRT task can deplete the capacity of its server, postponing the server deadline, which causes the server's priority to decrease.

As a consequence, an SRT task can potentially be blocked many times by every other task and several times on each resource. The worst-case interference time can be very high, whereas the average interference time will probably be much lower than the worst case. Moreover, since an SRT task can tolerate occasional deadline misses, there is no advantage to selecting the server's budget based on the worst-case computation time and worst-case interference time.

In order to assign the server parameters and then adjust them for minimizing the number of deadline misses, it is better to dynamically adjust the budget by using an online feedback mechanism like the one proposed by Abeni and Buttazzo [23] or the elastic model proposed by Buttazzo et al. [24].

\section{Conclusions ANd Future Work}

In this paper, we presented the Bandwidth Inheritance protocol, a novel scheduling discipline that allows the sharing of resources between real-time tasks in dynamic real-time systems. Coherently with the Resource Reservation approach, BWI provides some temporal isolation properties without requiring any a priori knowledge about the structure and the temporal behavior of the tasks. 
In addition, a schedulability analysis for HRT tasks has been developed and presented in this paper. This analysis is based on formal properties of the BWI protocol that are presented and proven in the paper.

As future work, we are currently analyzing new strategies for coping with SRT tasks. Roughly speaking, an SRT task that borrows interference time from another SRT task should give it back after some time. The idea is that every SRT task should be given a fair share of the processor over long intervals. Moreover, we are planning to combine feedback scheduling techniques [25], [24], [23] with BWI in order to adjust the parameters of the servers and minimize the number of missed SRT deadlines.

\section{ACKNOWLEDGMENTS}

This work was carried out while G. Lamastra and L. Abeni were PhD students at the Scuola Superiore Sant'Anna.

\section{REFERENCES}

[1] T. Ghazalie and T. Baker, "Aperiodic Servers in a Deadline Scheduling Environment," J. Real-Time Systems, vol. 9, 1995.

[2] M. Caccamo and L. Sha, "Aperiodic Servers with Resource Constraints," Proc. IEEE Real-Time Systems Symp., Dec. 2001.

[3] D. de Niz, L. Abeni, S. Saewong, and R. Rajkumar, "Resource Sharing in Reservation-Based Systems," Proc. IEEE Real-Time Systems Symp., Dec. 2001.

[4] L. Abeni and G. Buttazzo, "Integrating Multimedia Applications in Hard Real-Time Systems," Proc. 19th IEEE Real-Time Systems Symp., Dec. 1998.

[5] L. Sha, R. Rajkumar, and J.P. Lehoczky, "Priority Inheritance Protocols: An Approach to Real-Time Synchronization," IEEE Trans. Computers, vol. 39, no. 9, Sept. 1990.

[6] Z. Deng and J.W.S. Liu, "Scheduling Real-Time Applications in Open Environment," Proc. IEEE Real-Time Systems Symp., Dec. 1997.

[7] M. Spuri and G.C. Buttazzo, "Scheduling Aperiodic Tasks in Dynamic Priority Systems," J. Real-Time Systems, vol. 10, no. 2, 1996.

[8] B. Sprunt, L. Sha, and J. Lehoczky, "Aperiodic Task Scheduling for Hard-Real-Time Systems," J. Real-Time Systems, vol. 1, July 1989.

[9] J.K. Strosnider, J.P. Lehoczky, and L. Sha, "The Deferrable Server Algorithm for Enhanced Aperiodic Responsiveness in Hard-RealTime Environments," IEEE Trans. Computers, vol. 4, no. 1, Jan. 1995.

[10] G. Lipari, "Resource Reservation in Real-Time Systems," PhD dissertation, Scuola Superiore S. Anna, 2000.

[11] C.W. Mercer, S. Savage, and H. Tokuda, "Processor Capacity Reserves for Multimedia Operating Systems," Proc. IEEE Int'l Conf. Multimedia Computing and Systems, May 1994.

[12] R. Rajkumar, K. Juvva, A. Molano, and S. Oikawa, "Resource Kernels: A Resource-Centric Approach to Real-Time and Multimedia Systems," Proc. Fourth Real-Time Computing Systems and Applications Workshop, Nov. 1997.

[13] R. Rajkumar, L. Abeni, D.D. Niz, S. Gosh, A. Miyoshi, and S. Saewong, "Recent Developments with Linux/RK," Proc. Real Time Linux Workshop, Dec. 2000.

[14] H. Chu and K. Nahrstedt, "CPU Service Classes for Multimedia Applications," Proc. IEEE Int'l Conf. Mutimedia Computing and Systems, June 1999

[15] C.L. Liu and J.W. Layland, "Scheduling Algorithms for Multiprogramming in a Hard-Real-Time Environment," J. ACM, vol. 20, no. $1,1973$.

[16] M. Chen and K. Lin, "Dynamic Priority Ceilings: A Concurrency Control Protocol for Real-Time Systems," J. Real-Time Systems, vol. 2, 1990.

[17] T.P. Baker, "A Stack-Based Allocation Policy for Realtime Processes," Proc. IEEE Real-Time Systems Symp., Dec. 1990.

[18] K. Jeffay, "Scheduling Sporadic Tasks with Shared Resources in Hard-Real-Time Systems," Proc. 13th IEEE Real-Time Systems Symp., pp. 89-99, Dec. 1992.
[19] T.-W. Kuo and C.-H. Li, "Fixed-Priority-Driven Open Environment for Real-Time Applications," Proc. IEEE Real Systems Symp., Dec. 1999.

[20] L. Abeni, "Server Mechanisms for Multimedia Applications," Technical Report RETIS TR98-01, Scuola Superiore S. Anna, 1998.

[21] G. Buttazzo, Hard Real-Time Computing Systems: Predictable Scheduling Algorithms and Applications. Kluwer Academic, 1997.

[22] J.A. Stankovic, M. Spuri, K. Ramamritham, and G.C. Buttazzo, Deadline Scheduling for Real-Time Systems-EDF and Related Algorithms. Kluwer Academic, 1998.

[23] L. Abeni and G. Buttazzo, "Adaptive Bandwidth Reservation for Multimedia Computing," Proc. IEEE Real Time Computing Systems and Applications, Dec. 1999.

[24] G. Buttazzo, G. Lipari, M. Caccamo, and L. Abeni, "Elastic Scheduling for Flexible Workload Management," IEEE Trans. Computers, vol. 51, no. 3, pp. 289-302 Mar. 2002.

[25] C. Lu, J.A. Stankovic, G. Tao, and S.H. Son, "Design and Evaluation of a Feedback Control EDF Scheduling Algorithm," Proc. IEEE Real Time Systems Symp., Dec. 1999.

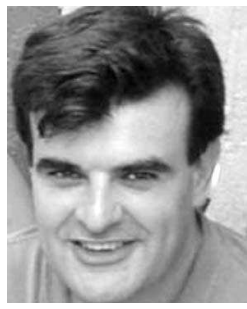

Giuseppe Lipari graduated in computer engineering from the University of Pisa in 1996 and received the $\mathrm{PhD}$ degree in computer engineering from the Scuola Superiore Sant'Anna in 2000. During 1999, he was a visiting student at the University of North Carolina at Chapel Hill, collaborating with Professor S.K. Baruah and Professor K. Jeffay on real-time scheduling. Currently, he is an assistant professor of operating systems with the Scuola Superiore Sant'Anna. His main research activities are in real-time scheduling theory and its application to real-time operating systems, soft real-time systems for multimedia applications, and component-based real-time systems. He is a member of the IEEE.

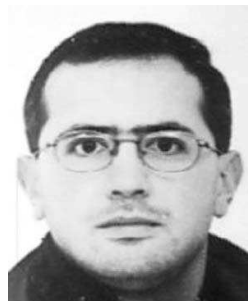

Gerardo Lamastra graduated with the degree in computer engineering from the University of Pisa, Italy, in 1996. In 2000, he received the PhD degree in computer engineering and real-time systems from the Scuola Superiore Sant'Anna, Pisa. In 2000, he joined Telecom Italia Lab, where he started working in the Internet Security Group (be-secure). In 2002, he received the SANS certification for System Forensics, Investigation and Response. His interests include: operating systems architecture and design, real-time systems, intrusion detection, and forensic analysis. He is a member of the IEEE.

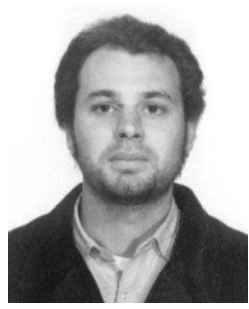

Luca Abeni graduated in computer engineering from the University of Pisa in 1998 and received the $\mathrm{PhD}$ degree in computer engineering from the Scuola Superiore Sant'Anna of Pisa, Italy, in 2002. During 2000, he was a visiting student at Carnegie Mellon University, working with Professor Ragunathan Rajkumar on resource reservation algorithms for real-time kernels. During 2002, he was a visiting student at the Oregon Graduate Institute, Portland, working on evaluating and enhancing the real-time performance of the Linux kernel. He currently works at Broadsat S.r.l., developing multimedia streaming solutions. He is a member of the IEEE.

For more information on this or any other computing topic, please visit our Digital Library at www.computer.org/publications/dlib. 